\title{
Multi-Color Electron Microscopy by Element-Guided Identification of Cells, Organelles, and Molecules
}

\author{
M. Scotuzzi ${ }^{1}$, J. Kuipers ${ }^{2}$, D. Wensveen ${ }^{1}$, P. de Boer ${ }^{2}$, N. Pirozzi $^{2}$, C. W. Hagen ${ }^{1}$, B. N. G. Giepmans ${ }^{2}$, \\ J. P. Hoogenboom ${ }^{1}$ \\ 1. Imaging Physics, Delft University of Technology, Delft, the Netherlands. \\ 2. UMC Groningen, Groningen, the Netherlands.
}

Electron microscopy (EM) is a powerful tool to study cellular complexity at nanometer resolution. Traditionally, EM images are acquired from small fields of view, but several new techniques allow routine acquisition of large 2D and 3D image stacks. However, in the resulting vast amount of greyscale data interpretation in terms of macromolecular functionality is difficult. With Correlative Light and Electron Microscopy (CLEM), fluorescence can be used to identify molecules in color[1] but the resolution gap with EM precludes accurate localization. The use of superresolution fluorescence microscopy techniques combined with CLEM[2,3] holds great promise but may be hard to achieve and the color palette limited. We are exploring novel approaches to achieve color-EM using the electron beam to add information about the specimen to the electron micrographs.

Large-scale EM is complemented with energy-dispersive-X-ray analysis (EDX) to allow EM analysis based on elemental composition. Different endogenous elements as well as exogenously introduced elements, including labels (gold- and cadmium-based nanoparticles) and stains, can be discriminated at ultrastructure resolution. This provides a wide palette of colors to paint the traditional grey-scale EM images for composition-based interpretation. Our proof-of-principle application of EM-EDX reveals that endocrine and exocrine vesicles exists in single cells in a Type-1 diabetes rat model (see Figure 1), highlighting how elemental mapping provides biomedical relevant information without bias. Broad application of EM-EDX will further allow experimental analysis on different kind of tissues, stains, and labels and thus brings nanometer-scale 'color-EM' as a promising tool to unravel molecular (de)regulation in biomedicine. 


\section{References:}

[1] P. de Boer, J. P. Hoogenboom, B. N. G. Giepmans, Nature Methods 12 (6) (2015), p. 503.

[2] Kopek, B.G., et al., Proc. Natl. Acad. Sci. U. S. A. 109 (2012), p. 6136.

[3] Johnson, E., et al., Scientific Reports 5 (2015).

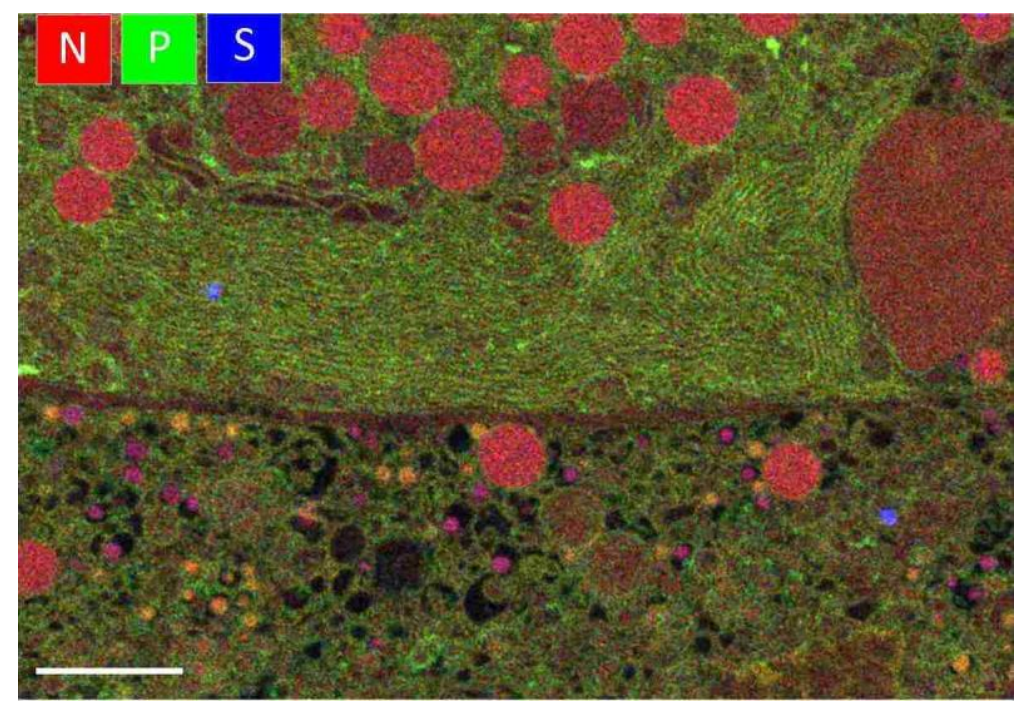

Figure 1. EDX analysis reveals single cells that contain exocrine zymogen granules with endocrine glucagon and insulin. Area from large-scale EM of rat pancreas tissue with parts of two cells with different granules. Colors result from overlay of EDX elemental maps for nitrogen $(\mathrm{N})$, phosphor $(\mathrm{P})$, and sulphur (S) as indicated. This allows identification of different granules: note the small purple and yellow granules, as well as the big red granule, all present in the same cell. Scalebar: $2 \mu \mathrm{m}$. 